\title{
Classificação de Sons Pulmonares com Redes Convolucionais e SVM
}

\author{
Guilherme L. Dias* Frederico L. de O. Mota** \\ Eduardo G. Ribeiro ${ }^{* * *}$ Bruno H. G. Barbosa ${ }^{* *}$ \\ Danton D. Ferreira ${ }^{* *}$ Ernesto L. Neto* \\ Leandro T. Gomes **** \\ * Departamento de Ciências da Saúde, Universidade Federal de Lavras, \\ Lavras, MG, (e-mail: guilherme.dias@estudante.ufla.br, \\ ernestolippi@outlook.com) \\ ** Departamento de Automática, Universidade Federal de Lavras, \\ Lavras, MG, (e-mail: frederico.mota@outlook.com, \\ brunohb@deg.ufla.br,danton@deg.ufla.br) \\ *** Departamento de Engenharia Elétrica e de Computação, Escola de \\ Engenharia de São Carlos, Universidade de São Paulo, São Carlos, \\ SP, (e-mail: eduardogr@usp.br) \\ **** Hospital Vaz Monteiro, Lavras, MG (e-mail: \\ gomes.leandroteixeira@gmail.com)
}

\begin{abstract}
Pulmonary auscultation has a great importance for clinical evaluation, and requires adequate environment and an experienced evaluator. This study aims to present an acquisition system to classify three classes of lung sounds (vesicular, wheezy and crackles) using two techniques. The first is based on Fast Fourier Transform (FFT), Genetic Algorithms and Support Vector Machine (SVM). It was possible to classify lung sounds with sensitivity of $87.5 \%$ and specificity of $93.7 \%$. The second technique uses the spectrograms with Convolutional Neural Networks (CNN) and has sensibility of $95.8 \%$ and the specificity of $87.5 \%$, and is able to classify wheezes and crackles with $96 \%$ and $92 \%$ of accuracy, respectively. Therefore, the SVM technique is superior in tracking and classifying lung sounds, at the cost of a higher rate of positive failures. In this wise, the digital stethoscope presented in this paper can be used to analyse lung sounds.

Resumo: A ausculta pulmonar tem grande importância para a decisão diagnóstica na prática médica e requer ambiente apropriado e um avaliador experiente. Este trabalho tem por objetivo apresentar um sistema de aquisição e classificação de três classes de sons pulmonares que são: vesiculares, sibilos e estertores, utilizando duas técnicas diferentes. Na primeira técnica, Algoritmos Genéticos são utilizados para selecionar as melhores componentes da Transformada Rápida de Fourier (FFT) dos sinais em conjunto com Máquinas de Vetores Suporte (SVM). Foi possível a classificação dos sons pulmonares com sensibilidade de $87,5 \%$ e especificidade de 93,7\%. A segunda técnica utiliza os espectrogramas dos sons e Redes Neurais Convolucionais (RNCs), nesse caso a sensibilidade foi de $95,8 \%$ e especificidade de $87,5 \%$, e é capaz de classificar os sibilos com $96 \%$ e estertores com $92 \%$ de acertos. Logo a RNC é superior em detectar e classificar os sons anormais, às custas de um maior índice de falsos positivos. Dessa forma, o estetoscópio digital apresentado pode ser empregado na análise de sons pulmonares.
\end{abstract}

Keywords: Lung sounds; Support Vector Machine; Computational Inteligence; Fast Fourier Transform; Convolutional Neural Network

Palavras-chaves: Sons Pulmonares; Máquina de Vetor de Suporte; Inteligência Computacional; Transformada Rápida de Fourier; Redes Neurais Convolucionais

\section{Introdução}

Segundo o Ministério da Saúde (2016) as doenças respiratórias crônicas representam cerca de $7 \%$ da mortalidade global, cerca de 4,2 milhões de óbitos anuais. Mais especificamente no Brasil, em 2011, elas foram a terceira causa de morte dentre as doenças crônicas não transmissíveis. Essas doenças acarretam limitações físicas, emocionais e intelectuais, gerando consequências negativas na qualidade de vida do paciente e de sua família. Ainda de acordo com o Forum of International Respiratory Societies (2017) cinco dessas doenças pulmonares são causas mais comumente associadas à morte que são: doença pulmonar obstrutiva crônica (DPOC), asma, infecção aguda do trato respiratório inferior, tuberculose e câncer de pulmão. As doenças respiratórias compõem cinco das trinta principais causas de morte no mundo, sendo a DPOC a terceira, infecção do trato respiratório inferior a quarta, câncer traqueal, brôn- 
quico e de pulmão a sexta, tuberculose a décima segunda e asma a vigésima oitava. Mais de 1 bilhão de pessoas sofrem de insuficiência respiratória aguda ou crônica. É muito perceptível a prevalência das patologias pulmonares e a alta mortalidade associada.

Uma previsão feita pela Organização Mundial da Saúde mostra que, em 2020, este conjunto de doenças pode ser a terceira causa de morte em todo mundo (Cao and Xiao, 2018). A análise destas doenças tem como ponto inicial o estudos dos sons respiratórios. Estes sons podem advir da boca, traqueia e pulmão. Os sons pulmonares são sinais fisiológicos aleatórios que ocorrem durante o processo de ventilação do sistema respiratório humano em contato com o ambiente externo (Wang et al., 2012). Estes sons são divididos em duas grandes classes, normais (vesiculares) e adventícios. A presença dos adventícios, como os sibilos e estertores, é um forte indicativo da existência de alguma doença no trato respiratório (Sovijarvi et al., 2000).

Os sibilos são sons adventícios que possuem como características ser contínuo e com uma duração mais elevada (normalmente maior que $100 \mathrm{~ms}$ ), incluindo pelo menos 10 vibrações sucessivas. Eles podem aparecer tanto durante a inspiração quanto na expiração. De uma perspectiva acústica, eles aparecem como oscilações sinusoidais com energia sonora na faixa de 100 a $1000 \mathrm{~Hz}$ e com harmônicos que excedem $1000 \mathrm{~Hz}$ em certas situações. Embora tipicamente presentes em doenças obstrutivas das vias aéreas, especialmente asma, não são indicativos definitivos de nenhuma doença em particular (Bohadana et al., 2014).

Os estertores tem como característica serem explosivos e aparecerem durante a inspiração. Eles podem ser distintos pela forma de onda, momento de aparição e tempo de duração (Reichert et al., 2008). Realizando uma comparação entre os estertores finos e grossos, tem-se como tempo de duração e frequência típicos de $5 \mathrm{msec}$ e $600 \mathrm{~Hz}$, respectivamente, para os finos, e $15 \mathrm{msec}$ e $350 \mathrm{~Hz}$ para os grossos (Bohadana et al., 2014). A presença de estertores grossos geralmente está relacionada com várias doenças (i.e. fibrose pulmonar intersticial, insuficiência cardíaca congestiva, pneumonia), assim como os estertores finos podem estar relacionados com bronquite crônica (Bohadana et al., 2014).

Devido à forte correlação existente entre a presença de sons adventícios e a detecção de doenças, é de grande importância realizar a investigação destes sons para que seja possível prevenir e identificar patologias no sistema respiratório mais facilmente e precocemente. Usualmente, a análise é feita por meio da técnica de diagnóstico chamada ausculta pulmonar. Esta técnica tem como características ser segura, não invasiva e não necessitar de grandes recursos financeiros para ser executada (Sarkar, M., 2015). Apesar disto, a efetividade da técnica é afetada pela qualidade técnica e experiencia do profissional que a está executando.

Com a expectativa de atenuar o erro e dependência humana, durante o procedimento de ausculta e diagnósticos, a utilização de técnicas de reconhecimento de padrões tem sido empregada para superar estas lacunas (Naves, R., 2016; Dias et al., 2018; Mota et al., 2017). Por exemplo, em Amaral et al. (2015), foi desenvolvido um sistema para tornar mais confiável a técnica de oscilação forçada (do inglês forced oscillation technique - FOT) na categorização do nível de obstrução das vias aéreas em pacientes com DPOC, basedo em três classificadores: o k-Nearest Neighbours (k-NN), Floresta Aleatória (RF) e Máquinas de Vetores de Suporte (SVM). Foi utilizada a curva ROC para mensurar o desempenho e os resultados alcançados foram superiores a 0,9 .

Máquinas de Vetores Suporte também foram usadas em (Jin et al., 2014) para classificação dos sons pulmonares, sendo que a Transformada de Gabor (um caso especial de Transformada Rápida de Fourier) foi utilizada na extração de características, obtendo resultados de acerto na classificação em torno de 97,7\% para sons inspiratórios e $98,8 \%$ para sons expiratórios.

A classificação de sete tipos sons pulmonares foi realizada em (Bardou et al., 2018) comparando três abordagens diferentes de machine learning. Duas abordagens utilizam três classificadores clássicos (SVM, k-NN e Mistura de Modelos Gaussianos) enquanto que a última foi baseada em Rede Neural Convolucional. Os resultados mostraram que a RNC foi superior aos outros métodos utilizados na classificação.

A proposta deste trabalho é, a partir da obtenção de um banco de dados próprio com sons pulmonares de pacientes reais adquiridos por um dispositivo de baixo custo desenvolvido pelo grupo de pesquisa, comparar duas técnicas de inteligência artificial, uma utilizando a Transformada Rápida de Fourier com SVM em conjunto com o Algoritmo Genético e outra utilizando Redes Neurais Convolucionais com os espectrogramas dos sons.

Na próxima seção será explicado o método proposto, detalhando o banco de dados, a extração de características e os classificadores desenvolvidos. Na Seção 3 serão apresentados os resultados encontrados e uma discussão sobre os métodos implementados. A Seção 4 apresenta as conclusões e direções futuras do trabalho.

\section{Materiais e Métodos}

\subsection{Banco de Dados}

As amostras utilizadas neste trabalho foram coletadas por meio de um protótipo de estetoscópio digital, no Hospital Vaz Monteiro, localizado em Lavras-MG, apresentado na figura 1 . O protótipo foi desenvolvido utilizando um microfone de eletreto acoplado ao diafragma do estetoscópio. O sinal é então amplificado por um circuito contendo dois amplificadores operacionais AD620AN e um filtro passa-baixa com frequência de corte de $10 \mathrm{kHz}$. O sinal é transmitido a um smartphone via cabo P2 e os dados são gravados por meio de um aplicativo, apresentado na figura 2, e salvos no formato WAV. A partir do banco de dados construído, técnicas de inteligência computacional são aplicadas a fim de realizar a classificação dos sons. Essas etapas são apresentadas na figura 3.

O banco de dados criado é composto por sons pulmonares, normais e adventícios, advindos de 8 pacientes, 4 saudáveis e 4 com patologias no sistema respiratório, que são amostrados a taxa de $8 \mathrm{kHz}$. Destes pacientes 


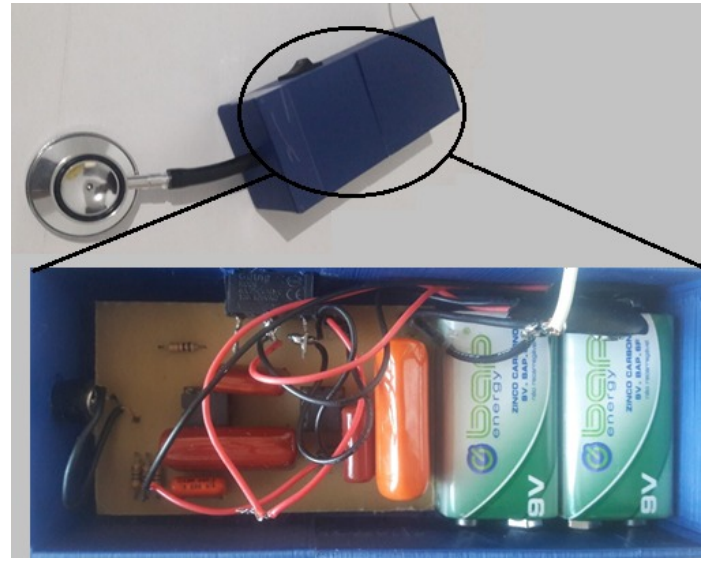

Figura 1. Protótipo do estetoscópio digital. Aspecto interno e externo.

3 são mulheres e 5 homens, com idade variando entre 22 e 86 anos, com média de 62,2 anos. Os sinais foram previamente normalizados com média nula e variância unitária. No total tem-se 400 sinais compostos por 320 amostras, obtidos a partir do janelamento dos sinais de cada paciente, conforme descrito em (Naves, R., 2016). Apesar de ser um número pequeno de pacientes, as formas de onda de cada som adventício é bastante característica e suas propriedades não variam significativamente entre pacientes, sendo que cada janela representativa da classe foi criteriosamente escolhida por um especialista. Os dados foram divididos em conjuntos de treino e teste do classificador proposto, obedecendo a fração de aproximadamente um quarto para teste. As tabelas 1 e 2 detalham o número de sinais de cada classe. A figura 2.1 apresenta exemplos de sons pulmonares de cada categoria no domínio do tempo e seu correspondente no domínio da frequência.

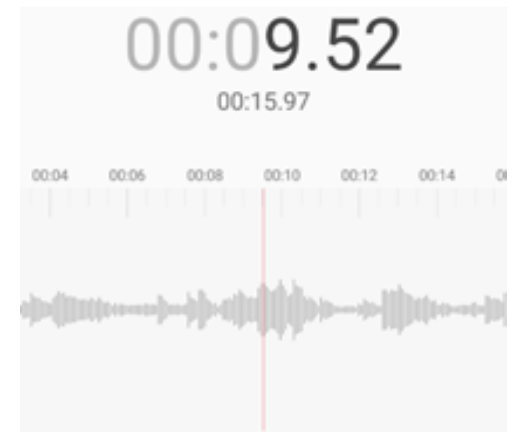

Figura 2. Aplicativo de aquisição para smartphone

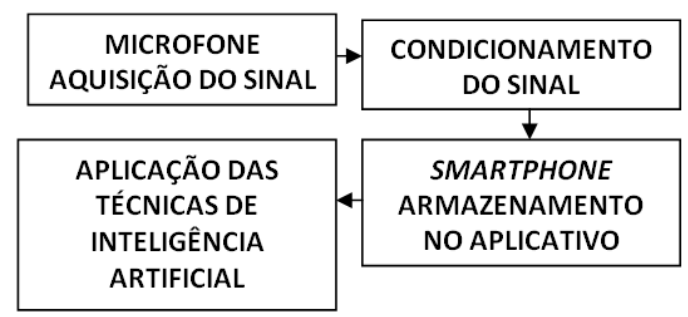

Figura 3. Esquemático da aquisição e tratamento dos dados
Tabela 1. Dados para treino.

\begin{tabular}{cc} 
Classe & $N^{\circ}$ de Sinais \\
\hline Vesicular & 152 \\
Sibilo & 76 \\
Estertor & 76 \\
\hline Total & 304 \\
\hline
\end{tabular}

Tabela 2. Dados para teste.

\begin{tabular}{cc} 
Classe & $\mathrm{N}^{\circ}$ de Sinais \\
\hline Vesicular & 48 \\
Sibilo & 24 \\
Estertor & 24 \\
\hline Total & 96 \\
\hline
\end{tabular}

\subsection{Classificador baseado em FFT, SVM e Algoritmo Genético}

2.2.1 Extração das características Para realizar a extração de características do banco de dados foi utilizada a Transformada Rápida de Fourier (em inglês Fast Fourier transform - FFT), algoritmo desenvolvido por (Cooley and Tukey, 1965). Este algoritmo é interessante pois utilizando a Transformada Discreta de Fourier para calcular todos os valores espectrais de $X$, são necessárias $N^{2}$ multiplicações complexas, ao passo que a FFT processa apenas $2 \mathrm{Nlog}_{2} \mathrm{~N}$ multiplicações complexas e, desta forma, permite que a Transformada de Fourier seja acessível e aplicável no processamento de sinais (Lathi, 2005).

Tendo em vista a classificação de sons pulmonares por meio dos coeficientes da série de Fourier realizada por (Biau et al., 2005; Dias et al., 2018), propõe-se o uso dos módulos da FFT dos sinais no método proposto para classificação destes. Excluindo-se a simetria conjugada do sinal resultante da aplicação do algoritmo, tem-se, por fim, um vetor com 160 características do sinal. A etapa seguinte visa a redução da dimensionalidade deste vetor.

2.2.2 Redução da dimensionalidade O Algoritmo Genético (Holland, 1992) é uma meta-heurística populacional baseada na Teoria da Evolução de Darwin e tem por objetivo encontrar a melhor solução (indivíduo), ou solução ótima, em problemas de otimização. A evolução da sua população depende das etapas de seleção dos mais aptos, trocas genéticas (cruzamentos) e mutação.

Neste trabalho, o Algoritmo Genético (AG) foi implementado de forma a encontrar o menor conjunto de características possível a ser utilizado em uma SVM que obtenha uma classificação satisfatória dos sons (técnica de seleção do tipo wrapper). Para isso, são criados indivíduos com $n$ posições, associados aos índices do vetor da FFT obtidos dos sinais de áudios dos sons pulmonares, podendo os valores variarem entre 1 e 160. A escolha dos indivídos é baseada no desempenho resultante do treinamento de SVMs com os $n$ índices definidos pelo indivíduo, nos seguintes problemas de classificação: $i$. vesicular versus adventício, $i i$. sibilios versus estertores (conforme apresentado na figura 5). As $n$ características do indivíduo são enviadas a uma SVM e o acerto da classificação (avaliação do indivíduo) é obtido em porcentagem, valor a ser maximizado pelo AG. A avaliação de cada indivíduo (conjunto de características) é realizada por meio da técnica de 

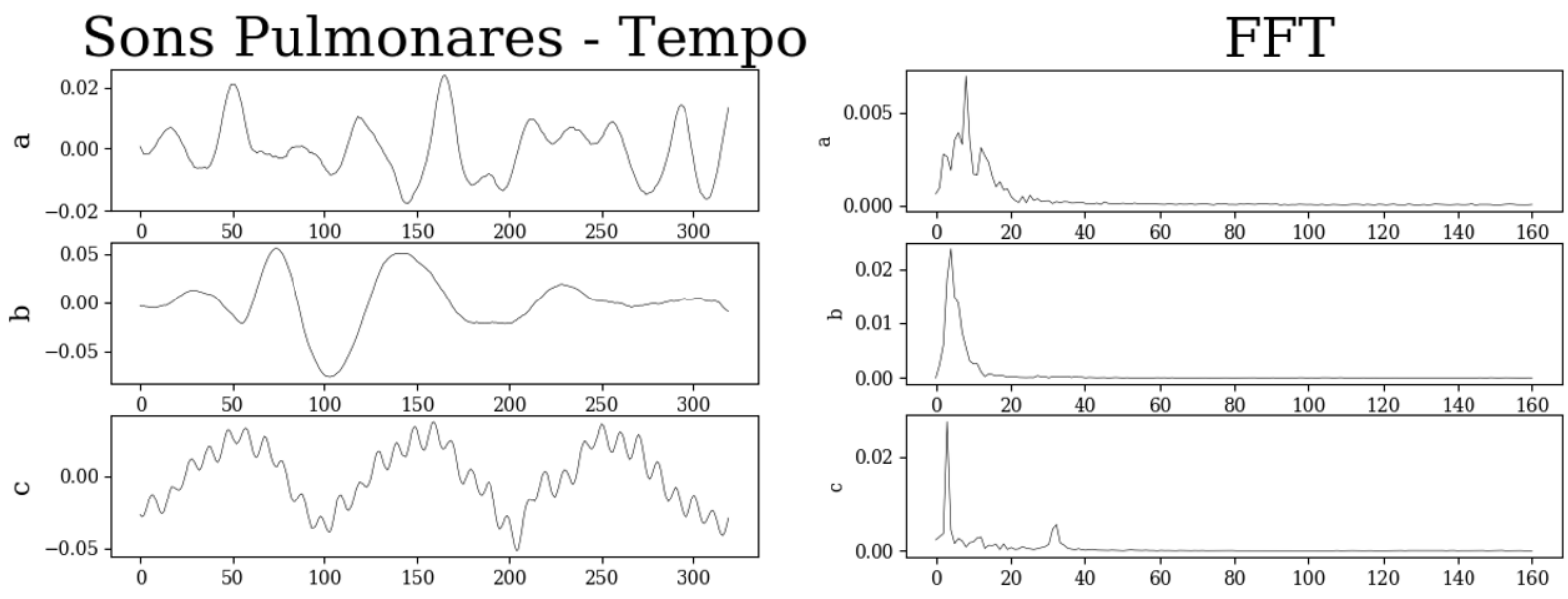

Figura 4. Sons Pulmonares: a - som vesicular, b - estertor, c - sibilo

validação cruzada do tipo $k$-fold, em dados de treinamento, com $k$ igual a cinco.

Após a avaliação, os indivíduos mais aptos são selecionados por meio da roleta viciada, cruzamentos são efetuados pelo método single point e a mutação do tipo swap é realizada, formando uma nova população. Desta forma, as características mais adequadas são evoluídas ao longo das gerações e, ao final da execução do AG, espera-se que o melhor conjunto de características seja encontrado.

A partir da realização de alguns testes, o AG foi executado diversas vezes com os melhores parâmetros encontrados (tamanho da população, número de gerações e probabilidades de cruzamento e mutação) e desempenhos muito próximos foram encontrados, sendo escolhido o melhor dentre eles. O critério de parada do AG é o número de gerações e os parâmetros utilizados são apresentados na tabela 3. A implementação do algoritmo foi realizada em Python versão 2.7.14, utilizando o Algoritmo Genético da biblioteca PyEvolve Genetics Algorithms 0.6.

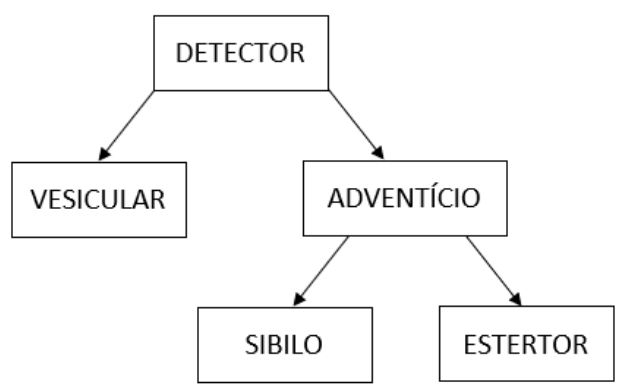

Figura 5. Fluxograma do método de classificação proposto com SVM

Tabela 3. Parâmetros do Algoritmo Genético para classificação entre Vesiculares e Adventícios (coluna 1), e entre Estertores e Sibilos (coluna 2)

\begin{tabular}{ccc} 
Parâmetros & 1 & 2 \\
\hline Taxa de cruzamento & 0,75 & 0,8 \\
\hline Taxa de mutação & 0,08 & 0,1 \\
\hline Número de gerações & 60 & 300 \\
\hline População & 90 & 160
\end{tabular}

2.2.3 Classificação Como alternativa às Redes Neurais Artificias, Máquinas de Vetores Suporte tem sido muito utilizadas em problemas de classificação. Sua teoria foi desenvolvida por (Vapnik, 1995) e se trata de uma técnica supervisionada de aprendizado de máquina. Seu treinamento baseia-se na busca de um hiperplano ótimo de separação entre classes em um espaço multidimensional. Os chamados vetores suporte, que se posicionam no limite das classes, servirão de base para o cálculo deste hiperplano ótimo.

O seguinte par $\left(\mathbf{w}^{*}, b^{*}\right)$ define o hiperplano ótimo:

$$
\mathbf{w}^{*}=\sum_{i=1}^{n} \alpha_{i}^{*} y_{i} x_{i}
$$

$$
b^{*}=\frac{1}{2}\left[\max \left\{i \mid y_{i}=-1\right\}(u)+\min \left\{i \mid y_{i}=+1\right\}(u)\right],
$$

em que $\alpha_{i}$ representam multiplicadores de Lagrange obtidos no processo de otimização quadrática, $x_{i}$ e $y_{i}$ representam o conjunto de dados de treinamento, sendo $u=\left(\mathbf{w}^{*} \cdot \mathbf{x}_{i}\right)$. Após a definição dos parâmetros na etapa de treinamento, a fase de teste se baseia na classificação de amostras $\mathbf{x}$ por meio da seguinte equação:

$$
g(x)=\operatorname{sgn}(f(x))=\operatorname{sgn}\left(\sum_{x_{i} \in S V} \alpha_{i}^{*} y_{i} \mathbf{x}_{i} \cdot \mathbf{x}+b^{*}\right),
$$

em que o resultado de $g(x)$ pode ser +1 ou -1 se o valor de $f(x)$ for $>0$ ou $<0$, respectivamente.

A SVM proposta neste trabalho possui kernel linear e soft-margin igual a 1 (Vapnik, 1995). O treinamento da SVM foi realizado em Python, biblioteca Scikit-Learn Machine Learning in Python 0.18.2

\subsection{Classificador baseado em Redes Neurais Convolucio- nais}

As Redes Neurais Convolucionais (RNC) (LeCun et al., 1989) são um tipo especializado de rede neural capaz de processar dados que possuem estrutura espacial, 
como imagens. De maneira simplista, as RNCs são redes neurais que utilizam a operação de convolução no lugar da multiplicação matricial em pelo menos uma de suas camadas (Goodfellow et al., 2016).

Do ponto de vista biológico, as RNCs foram inspiradas pelo funcionamento do córtex visual, que possui pequenas regiões com células especializadas em cada área do campo visual. O experimento desenvolvido no trabalho de Hubel and Wiesel (1962) mostrou que neurônios individuais eram ativados somente na presença de padrões específicos, tais como bordas em orientações determinadas. Esta ideia de componentes especializados pode ser encontrada na fundamentação das RNCs.

Para valer-se da estrutura espacial das imagens $I$, as redes são ajustadas de modo que os filtros convolutivos têm seus pesos atualizados por meio da retropropagação do erro. Estes filtros são representados por $K$ na seguinte representação do operador de convolução:

$$
S[i, j]=K[i, j] * I[i, j]=\sum_{m} \sum_{n} I[i-m, j-n] K[m, n] .
$$

em que $i$ e $j$ são os índices das linhas e colunas, respectivamente, da imagem e filtro.

De forma geral, pode-se entender as RNCs como a conjunção de camadas convolucionais, que atuam na extração automática de características relevantes da imagem e camadas totalmente conectadas, que utilizam estas características para gerar uma regra de classificação, ou regressão.

Alguns elementos adicionais podem ser considerados na construção da arquitetura da rede para que esta possua maior capacidade de generalização e maior velocidade de treinamento. Alguns desses elementos foram utilizados na arquitetura proposta e são descritos a seguir.

2.3.1 Unidade Linear Retificada (ReLU) A unidade linear retificada (Jarrett et al., 2009) é a atual recomendação de função de ativação em RNCs (Goodfellow et al., 2016), primeiramente por causar a diminuição no tempo de treinanemento da rede, visto que funções sigmoidais saturam, ao passo que a ReLU é a função identidade para valores positivos (Ponti and da Costa, 2018). Outro ponto primordial é o fato de a ReLU evitar o problema de dissipação do gradiente.

2.3.2 Subamostragem (Pooling) A operação de subamostragem ajuda a tornar as representações invariantes a pequenas translações da entrada. Esta propriedade pode ser útil em situações em que a ocorrência de uma característica é mais importante que sua localização (Goodfellow et al., 2016). A mais tradicional forma de subamostragem é o pooling máximo (Zhou and Chellappa, 1988), em que o valor máximo de uma janela é o único mantido.

2.3.3 Desligamento (Dropout) Dropout é uma técnica para minimizar o sobre-ajuste proposta por Srivastava et al. (2014). O procedimento consiste em desativar, com uma probabilidade $p$, a ativação dos neurônios das camadas totalmente conectadas na fase de treinamento. Ao desativar alguns neurônios a complexidade das funções
Tabela 4. Parâmetros de treinamento

\begin{tabular}{cc}
\hline Função Custo & Entropia Cruzada \\
Algoritmo de Otimização & RMSprop \\
Taxa de Aprendizagem & $10^{-4}$ \\
Inicialização dos pesos & Xavier \\
Inicialização dos biases & 0 \\
Número máximo de épocas & 15 \\
Tamanho do batch & 16 \\
Ativação das camadas & ReLU \\
Ativação da camada de saída & Softmax \\
\hline
\end{tabular}

é reduzida uma vez que menos ativações são utilizadas. Ou seja, dropout funciona também como uma forma de regularização.

2.3.4 Normalização por lotes (Batch Normalization) A normalização por lotes é uma técnica usada para melhorar o desempenho e aumentar a estabilidade de redes neurais. A ideia é normalizar as entradas de cada camada de tal modo que tenham média zero e variância unitária, assim como o padrão normalmente seguido para a normalização da entrada da rede. A justificativa matemática para o funcionamento e benefícios da normalização por lotes pode ser encontrada em (Ioffe and Szegedy, 2015).

Os valores utilizados para treinamento e as camadas utilizadas na rede estão escritas nas tabelas 4 e 5 .

Para treinamento, o algoritmo de otimização utilizado é o RMSprop (Tieleman and Hinton, 2012), que atualiza os valores dos parâmetros com base na seguinte equação:

$$
W_{t+1}=W_{t}-\frac{\eta}{\sqrt{\nu_{t}+\epsilon}} \nabla E(W)
$$

em que $\nu_{t}$ representa uma taxa de decaimento exponencial do quadrado dos gradientes, $\eta$ é a taxa de aprendizagem, $\epsilon$ é um valor para evitar divisão por zero e $\nabla E(W)$ é o gradiente da função custo.

A rede recebe como entrada um espectrograma do som pulmonar no formato $(496 \times 369 \times 3)$, em que os dois primeiros valores referem-se às dimensões de comprimento e altura. O terceiro valor é a quantidade de canais da imagem, ou seja, vermelho, verde e azul - sistema de cores RGB.

Tabela 5. Dimensões de saída e quantidade de parâmetros das camadas

\begin{tabular}{c|c|c}
\hline Camada & Dimensões da saída & Parâmetros \\
\hline Camada Convolucional & $(246,183,64)$ & 4864 \\
MaxPooling & $(123,91,64)$ & 0 \\
Batch Normalization & $(123,91,64)$ & 256 \\
Camada Convolucional & $(61,45,128)$ & 73856 \\
MaxPooling & $(30,22,128)$ & 0 \\
Batch Normalization & $(30,22,128)$ & 512 \\
Camada Convolucional & $(30,22,128)$ & 147584 \\
Camada Convolucional & $(30,22,128)$ & 147584 \\
Camada Convolucional & $(30,22,128)$ & 147584 \\
MaxPooling & $(15,11,128)$ & 0 \\
Batch Normalization & $(15,11,128)$ & 512 \\
Flattenning & $(21120)$ & 0 \\
Dropout & $(21120)$ & 0 \\
Totalmente Conectada & $(128)$ & 2703488 \\
Saída & $(3)$ & 387 \\
\hline
\end{tabular}


Tabela 6. Matriz de confusão para dados de teste - abordagem com SVM, AG e FFT.

\begin{tabular}{c|c|c|c|}
\multicolumn{1}{c}{} & \multicolumn{1}{c}{ Vesicular } & \multicolumn{1}{c}{ Sibilo } & \multicolumn{1}{c}{ Estertor } \\
\cline { 2 - 4 } Vesicular & $0,94(45)$ & $0,02(1)$ & $0,04(2)$ \\
\cline { 2 - 4 } Sibilo & $0,08(2)$ & $0,88(21)$ & $0,04(1)$ \\
\cline { 2 - 4 } Estertor & $0,16(4)$ & $0,08(2)$ & $0,76(18)$ \\
\cline { 2 - 4 } & &
\end{tabular}

Os espectrogramas foram gerados a partir de sinais contendo 320 amostras obtidas a $8 \mathrm{kHz}$, conforme protocolo seguido na metodologia AG+SVM. Foram utilizadas 256 amostras para cálculo do espectro e 128 como overlapping entre janelas, de acordo com o padrão da função utilizada em Python. O resultado é um espectrograma contendo apenas uma coluna com eixo temporal no intervalo 0,008 $0.024 \mathrm{~s}$ e eixo de frequências na faixa $0-4000 \mathrm{~Hz}$. Por fim, para que possa ser introduzido na rede, o gráfico de dimensões $640 \times 480$ é reduzido às dimensões $496 \times 369$ após a retirada de seus eixos e da área sem informação relevante para a rede (bordas em branco). O resultado é ilustrado na figura 6 .

A rede foi implementada em Python por meio do framework Keras com backend em Tensorflow. O hardware utilizado possui processador Intel Core i7-7500U 2,9GHz e placa de vídeo Nvidia GeForce 940MX com capacidade de compilação de algoritmos de aprendizagem profunda.

\section{Resultados e discussão}

O algoritmo genético quando limitado a 90 características para classificação entre sons vesiculares e adventícios selecionou 69 características. Já para classificação entre sibilos e estertores quando limitado à 160 características escolheu 72 delas. Percebeu-se ainda durantes os testes para classificar vesicular versus adventício que um número maior de características possíveis associado a mais gerações criava maior confusão entre as classes, ao ponto que um número menor de gerações, no caso 60, quando comparado à classificação dos sibilos versus estertores em que se utilizou 200 gerações, foi melhor.

Os resultados de teste, em formato de matriz de confusão, para as SVMs treinadas com as componentes da FFT selecionadas por AGs, são apresentados na tabela 6 . Nesta tabela são apresentados os acertos de classificação das duas SVMs treinadas, de acordo com o fluxograma de classificação dos sons apresentado na figura 5, ou seja, uma SVM aplicada na classificação entre sons vesiculares e adventícios e a outra SVM que classifica entre estertores e sibilos.

Como pode ser observado na tabela 6 , ocorre confusão maior entre as classes vesiculares e estertores do que entre vesiculares e sibilos. Além disso, o sistema proposto deixa de classificar como adventícios $8 \%$ dos sibilos e $16 \%$ dos estertores. Este desempenho pode ser indesejável considerando a aplicação deste sistema em um processo de triagem em hospitais, pois a taxa de falso negativo (quando o som apresenta anomalia mas é classificado como normal) é mais problemática do que o contrário, quando não há anormalidade no som mas ele é classificado como adventício (falso positivo).

Considerando que sibilos e estertores são sinais adventícios, portanto anormais ou patológicos, e que os vesicu-
Tabela 7. Matriz de confusão para dados de teste - abordagem com RNC.

\begin{tabular}{c|c|c|c|}
\multicolumn{1}{c}{} & \multicolumn{1}{c}{ Vesicular } & \multicolumn{1}{c}{ Sibilo } & \multicolumn{1}{c}{ Estertor } \\
\cline { 2 - 4 } Vesicular & $0,88(42)$ & $0,08(4)$ & $0,04(2)$ \\
\cline { 2 - 4 } Sibilo & $0,04(1)$ & $0,96(23)$ & $0,00(0)$ \\
\cline { 2 - 4 } Estertor & $0,04(1)$ & $0,04(1)$ & $0,92(22)$ \\
\cline { 2 - 4 } & &
\end{tabular}

lares são normais, então a sensibilidade é de $87,5 \%$ e a especificidade de 93,7\%. O algoritmo apresenta melhores resultados para confirmar se o som normal de fato é normal, mas tem baixo poder de classificação entre as alterações, principalmente pela taxa de falso negativo presente.

Utilizando a abordagem com RNC tem-se a matriz confusão em dados de teste apresentada na tabela 7 . Esta abordagem apresenta melhores resultados no que tange o índice de falsos negativos, apenas $4 \%$ para cada uma das classes de adventícios (sibilos e estertores). No entanto, apresenta um maior índice de falsos positivos, $12 \%$ dos sons vesiculares são classificados adventícios, sendo que $8 \%$ são confundidos com sibilos e $4 \%$ com estertores. Para a classificação dos sibilos ocorre confusão de $4 \%$ com vesiculares, e os estertores são confundidos $8 \%$ das vezes, sendo $4 \%$ com vesiculares e outros $4 \%$ com sibilos. Portanto, os sibilos são classificados com $96 \%$ de acertos e os estertores com $92 \%$ de acertos, bem acima dos $88 \%$ e $76 \%$ de acerto da abordagem com SVM. Nesse caso a sensibilidade é de $95,8 \%$ e a especificidade de $87,5 \%$.

Considerando a aplicação de um equipamento para triagem de pacientes em um hospital, a escolha de um classificador com mais sensibilidade é mais interessante, podendo definir melhor a avaliação inicial realizada pelo serviço de enfermagem, que associada à outros critérios clínicos poderão determinar de forma mais acurada a gravidade do paciente. Doenças prevalentes do pronto socorro como a asma, a insuficiência cardíaca, pneumonias, doença pulmonar obstrutiva crônica poderiam ser melhor identificadas ou ter sua gravidade melhor avaliada pela avaliação do dispositivo. Além disso, com índices de acertos de $96 \%$ e $92 \%$ nas classes adventícias, é preferível implementar o classificador RNC, apesar de um custo computacional mais elevado do mesmo. Pode ser considerado satisfatório também uma classificação de sons vesiculares com $88 \%$ de acerto.

A obtenção de classificadores com índices de acerto satisfatórios comprova que o sistema de aquisição de sons apresentado (estetoscópio digital) é também válido, conseguindo disponibilizar sons pulmonares passíveis de serem analisados por sistemas de reconhecimento de padrões. Ademais, trata-se de um sistema de baixo custo monetário.

\section{Conclusão}

Este trabalho apresentou um sistema de aquisição de sons pulmonares de baixo custo (estetoscópio digital) capaz de obter sons com qualidade suficiente para serem posteriormente classificados por um sistema inteligente de reconhecimento de padrões.

Duas abordagens foram apresentadas para classificação de três tipo de sons: vesiculares (normais) e os adventícios estertores e sibilos. A primeira abordagem de classificação dos sons pulmonares proposta utilizou FFT, 


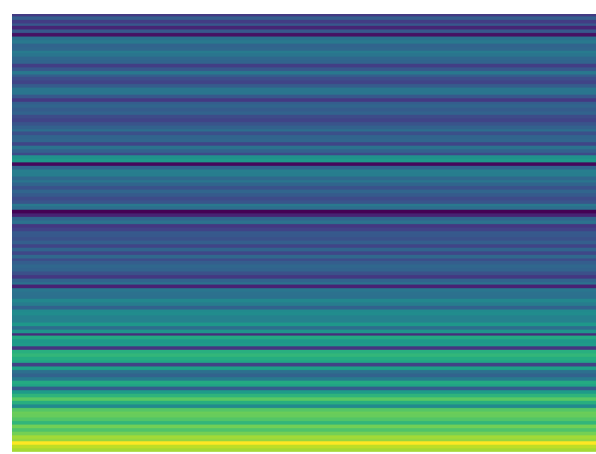

Sibilo

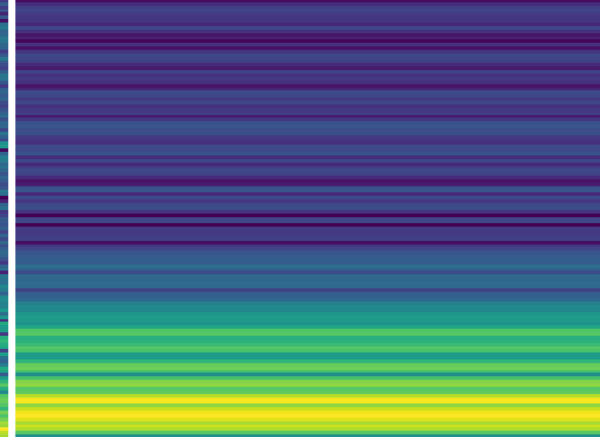

Estertor

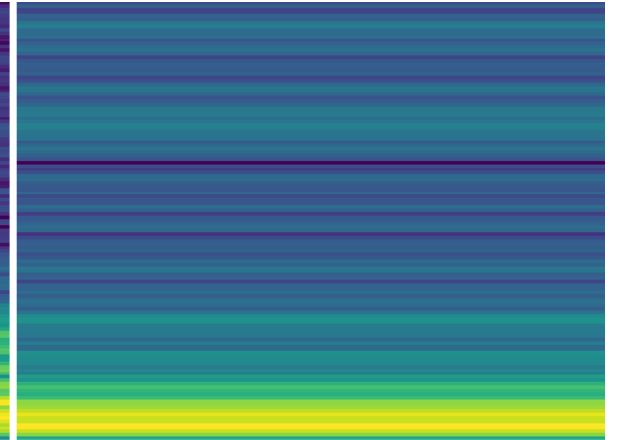

Vesicular

Figura 6. Exemplos de espectrogramas utilizados como entrada pela rede convolucional

SVM e o Algoritmo Genético, obtendo um alto valor de especificidade $(93,7 \%)$, mas com um índice elevado de falsos negativos (13\%). Uma segunda abordagem foi proposta utilizando as Redes Neurais Convolucionais, apresentando uma maior sensibilidade (95,8\%), sendo mais indicada para fins de triagem e detecção de patologia. Além disso, as RNCs classificaram os sons sibilos e estertores com $96 \%$ e $92 \%$ de acerto, respectivamente.

Como trabalhos futuros, pretende-se aumentar o banco de dados com mais pacientes e outros tipos de sons pulmonares, como o atrito pleural. Ademais, almeja-se implementar o sistema de reconhecimento de padrões em hardware embarcado.

\section{Agradecimentos}

Agradecimento ao CNPq (Conselho Nacional de Desenvolvimento Científico e Tecnológico) e à FAPEMIG (Fundação de Amparo a Pesquisa do Estado de Minas Gerais) pelos apoio financeiro.

\section{Referências}

Amaral, J.L., Lopes, A.J., Faria, A.C., and Melo, P.L. (2015). Machine learning algorithms and forced oscillation measurements to categorise the airway obstruction severity in chronic obstructive pulmonary disease. Computer methods and programs in biomedicine, 118(2), 186-197.

Bardou, D., Zhang, K., and Ahmad, S.M. (2018). Lung sounds classification using convolutional neural networks. Artificial intelligence in medicine, 88, 58-69.

Biau, G., Bunea, F., and Wegkamp, M.H. (2005). Functional classification in hilbert spaces. IEEE Transactions on Information Theory, 51(6), 2163-2172.

Bohadana, A., Izbicki, G., and Kraman, S.S. (2014). Fundamentals of lung auscultation. New England Journal of Medicine, 370(8), 744-751.

Cao, X.Y. and Xiao, S.Y. (2018). Chronic lung disease, lung regeneration and future therapeutic strategies. $\mathrm{Ch}$ ronic diseases and translational medicine, 4(2), 103-108.

Cooley, J.W. and Tukey, J.W. (1965). An algorithm for the machine calculation of complex fourier series. Mathematics of computation, 19(90), 297-301.

Dias, G.L., de Oliveira Mota, F.L., Barbosa, B.H.G., Ferreira, D.D., and Neto, E.L. (2018). Classificador de sons pulmonares: Uma abordagem baseada em fft e maquina de vetor de suporte. Congresso Brasileiro de Automática.

Forum of International Respiratory Societies (2017). The Global Impact of Respiratory Disease. European Respiratory Society.

Glorot, X. and Bengio, Y. (2010). Understanding the difficulty of training deep feedforward neural networks. In Proceedings of the thirteenth international conference on artificial intelligence and statistics, 249-256. Pascal2, Sardinia, Italy.

Goodfellow, I., Bengio, Y., Courville, A., and Bengio, Y. (2016). Deep learning, volume 1. MIT Press, Cambridge, USA.

Holland, J.H. (1992). Adaptation in natural and artificial systems: an introductory analysis with applications to biology, control, and artificial intelligence. MIT press.

Hubel, D.H. and Wiesel, T.N. (1962). Receptive fields, binocular interaction and functional architecture in the cat's visual cortex. The Journal of physiology, 160(1), 106-154.

Ioffe, S. and Szegedy, C. (2015). Batch normalization: Accelerating deep network training by reducing internal covariate shift. arXiv preprint arXiv:1502.03167.

Jarrett, K., Kavukcuoglu, K., LeCun, Y., et al. (2009). What is the best multi-stage architecture for object recognition? In Computer Vision, 2009 IEEE 12th International Conference on, 2146-2153. IEEE, Kyoto, Japan.

Jin, F., Sattar, F., and Goh, D.Y. (2014). New approaches for spectro-temporal feature extraction with applications to respiratory sound classification. Neurocomputing, $123,362-371$.

Lathi, B.P. (2005). Linear systems and signals. A systematic review and meta-analysis. Oxford University Press, New York.

LeCun, Y. et al. (1989). Generalization and network design strategies. Connectionism in perspective, 143-155.

Ministério da Saúde (2016). Boletim epidemiológico - perfil da morbimortalidade por doenças respiratórias crônicas no brasil, 2003 a 2013. Secretaria de Vigilância em Saúde, 19(19).

Mota, F.L.O., Dias, G.L., Godinho, E.R., Barbosa, B.H.G., Ferreira, D.D., and Neto, E.L. (2017). Detecção de anormalidades em sons pulmonaresbaseada em fft e máquinas de vetores suporte. Congresso Brasileiro de Inteligência Computacional.

Naves, R., Barbosa, B. H. G. e Ferreira, D.D. (2016). Classification of lung sounds using higher-order statistics: A 
divide-and-conquer approach., volume 129. Computer Methods and Programs in Biomedicine.

Ponti, M.A. and da Costa, G.B.P. (2018). Como funciona o deep learning. arXiv preprint arXiv:1806.07908.

Reichert, S., Gass, R., Brandt, C., and Andrès, E. (2008). Analysis of respiratory sounds: state of the art. Clinical medicine. Circulatory, respiratory and pulmonary medicine, 2, CCRPM-S530.

Sarkar, M., Madabhavi, I. e Niranjan, N. (2015). Auscultation of the respiratory system. Annals of thoracic medicine., 10(3), 158-168.

Sovijarvi, A., Dalmasso, F., Vanderschoot, J., Malmberg, L., Righini, G., and Stoneman, S. (2000). Definition of terms for applications of respiratory sounds. European Respiratory Review, 10(77), 597-610.

Srivastava, N., Hinton, G., Krizhevsky, A., Sutskever, I. and Salakhutdinov, R. (2014). Dropout: a simple way to prevent neural networks from overfitting. The Journal of Machine Learning Research, 15(1), 1929-1958.

Tieleman, T. and Hinton, G. (2012). Lecture 6.5-rmsprop: Divide the gradient by a running average of its recent magnitude. COURSERA: Neural networks for machine learning, 4(2), 26-31.

Vapnik, V. (1995). The Nature of Statistical Learning Theory. Springer-Verlag, New York.

Wang, B., Miao, L., Dong, H., and Zheng, Z. (2012). The research of lung sound signals based on cepstrum analysis. In 2012 International Conference on Biomedical Engineering and Biotechnology, 934-938. IEEE.

Zhou, Y.T. and Chellappa, R. (1988). Computation of optical flow using a neural network. In IEEE International Conference on Neural Networks, volume 1998, 71-78. IEEE, Anchorage, USA. 